\title{
AMENDER: an Attentive and Aggregate Multi-layered Network for Dataset Recommendation
}

\begin{abstract}
In this paper, we study the problem of recommending the appropriate datasets for authors. Comparing to existing studies that retrieve datasets passively from queries, recommending datasets actively based on author profiles can cause their attention on previously unknown datasets. In this paper, we implement the dataset recommendation system by inferring the proximity between authors and datasets, on top of a three-layered network, composed by authors, papers and datasets, respectively. To link author-dataset semantically by taking advantage of the rich content information of papers in the intermediate paper layer, we design an attentive and aggregate multi-layered network learning model. The aggregation is for efficiently integrating the intra-layer information of paper content and citations, while the attention is used for explicitly coordinating authors at the toplayer and datasets at the bottom-layer in the semantic space learned from papers in the intermediate layer. We test our model in a real scholarly dataset, and the experimental study demonstrates the superiority of our method compared with the solutions that extend existing models to our problem.
\end{abstract}

Index Terms-Recommendation systems, Multi-layered network, Network embeddinng

\section{INTRODUCTION}

In modern research communities, researchers are living and breathing with data. State-of-the-art studies, such as BERT [?], RESNET [?] and Transformer-XL [?], made themselves convincing by presenting their work's impact on typical benchmark datasets. However, with the growing volume and diversity of publicly available datasets, researchers are spending an increasing amount of time on managing and exploring useful datasets [?], even from well-organized and domain-specific dataset repositories. For example, when a researcher wants to discover a new field or a newly enrolled Ph.D. student starts a new project, the majority of the warm-up time is to find the most reliable and well-studied datasets in the related field [?], [?]. Luckily, a few organizations and institutes, such as Google, Elsevier and Mendeley, have already offered their solutions: several dataset search engines are being available for researchers from all around the world [?], [?], [?]. However, these dataset search engines only provide general dataset searching service for ordinary researchers, while none of them provide personalized recommendation for users.

In this paper, we aim at building a customized dataset recommendation model for researchers by mining their interactions and their semantics from publications. As presented in Figure ??, our problem is depicted as a three-layer network, including author, paper and dataset layers. The inter-layer links are formed via authorship between authors and papers, and by dataset citations between papers and datasets. At the paper layer, papers are linked by citations among them. At

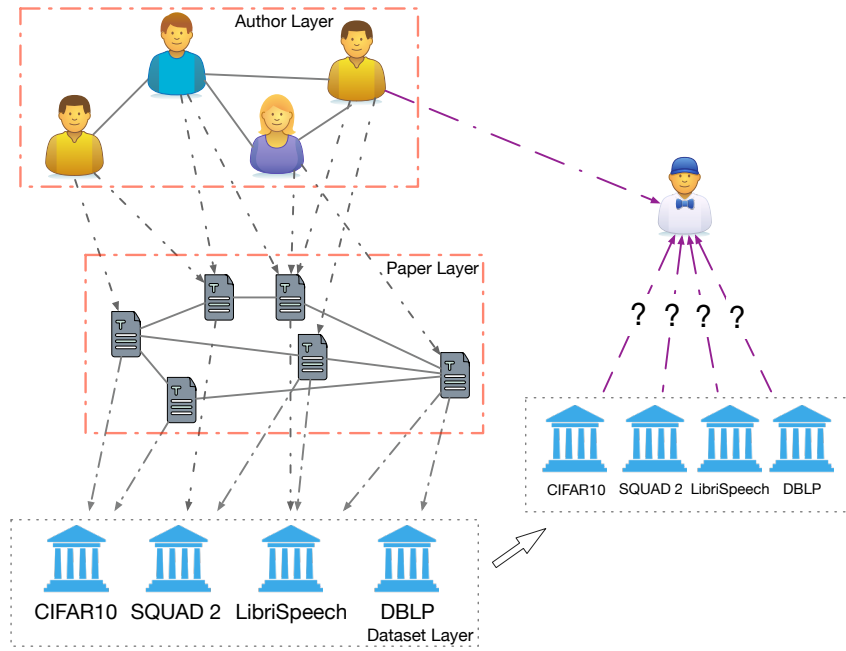

Fig. 1: Given the three-layer network of Author, Paper, and Dataset, we target on inferring the research interests of a user and recommend the appropriate datasets to be used.

the researcher layer, people can be linked by their academic connections, e.g., coauthor-ship, co-affiliation interactions, follower/followee on ResearchGate or GoogleScholar. For a user, shown in the right side of Figure ??, we want to infer his preference to the existing datasets. As it has been investigated in [?], [?], [?], using embedding techniques to model social relations is the new fashion and have proved their effectiveness on improving recommending results. However, unlike previous social recommendation methods that focus on using a single layer social relation at the user side to infer the links with the other side of a bipartite graph (items), we model the interactions at the top two layers (authors and papers), and infer the links with the bottom layer (datasets) in the threelayered network.

However, the special nature of our problem also prevents the direct adoption of existing heterogeneous network based recommendation models. Embedding approaches designed for heterogeneous networks [?], [?] or multi-layered network [?] do consider the node types and represent nodes by lowdimensional vectors. However, meta-path based methods [?] fuse the heterogeneous information while ignoring the relationship between homogeneous nodes, like author-author and paper-paper relationship in our problem. Moreover, the learned embeddings may not be suitable for the recommendation tasks, considering the two significant challenges in dataset recommendation, extreme data sparsity and cold start [?]. 
In the context of recommender systems, task-guided, pathaugmented, and semantic-aware heterogeneous network embedding methods are being proposed (e.g., [?], [?], [?], [?]). However, they are designed for bipartite networks (with two layers). In contrast, we would like to learn from a three-layer network and fuse the content information of nodes and the intra-layer/inter-layer interactions.

To measure the proximity of authors and datasets by their semantic representations, we propose AMENDER, a network learning model for dataset recommendation. The general idea of our solution is, 1) learn the paper representations in the middle layer by intensively using both their contents and citation relations; 2) then, coordinate authors at the top-layer and datasets at the bottom-layer in the semantic space by using the most representative papers; 3 ) for the recommendation purpose, author-dataset ranking loss is taken as the objective function defined on the author and dataset representation learned in 2). For objects at the same layer, like papers, the aggregation is used for integrating the paper-layer structure and content information to produce paper representations. Since the influence of intra-layer objects will propagate iteratively, aggregation can obtain the information from its further neighborhood. Also, the aggregation operator is computationally efficient for learning from large networks. The multi-head attention mechanism strengthens the model for capturing important information from cross-layer neighborhood. As a result, authors and datasets representation are adjusted to take different influence from papers by using multi-head attention for inter-layer interactions. Finally, authors at the top-layer and datasets at the bottom-layer are represented in the semantic space by making the usage of the rich content information of papers in the intermediate paper layer. To mitigate the impact of name ambiguity in scholarly data [?], we adopt the adversarial personalized ranking (APR) to stabilize our training. We demonstrate the effectiveness of our model on the collected scholarly dataset. The extensive experiments show that our proposed AMENDER model delivers more accurate recommendation results than the other solutions that adapt the existing models to dataset recommendation problem.

Here we briefly summarized our contributions as follows:

- We studied the author-dataset recommendation problem in real scholarly data, which can benefit the whole academic community.

- We design a model to measure the semantic proximity of authors and datasets in the three-layer network by integrating the paper content information and the intralayer/inter-layer interactions.

- We conduct extensive evaluations and demonstrate the effectiveness of our model on several scholarly recommendation tasks. The results show that AMENDER outperforms other baseline methods over a significant margin.

\section{RELATED WORK}

Due to the characteristics of relational data, many scholarly data studies use network representation learning approaches to learn the features for authors and papers in scholarly data. Recommendation methods for scholarly data also benefit from representation learning approaches, which modeled the scholarly data as heterogeneous information networks (e.g., [?], [?]), and adopt meta-path based representation learning for effective recommendation.

\section{A. Representation Learning for Scholarly Data}

Introducing representation methods to discover the features in scholarly data has caused increasing attention. General network representation methods can deal with the feature learning for scholarly data [?], [?]. However, they map all nodes into the same representation space and ignore the characteristics of different object types. Network representation learning methods for heterogeneous network can discriminate different types for scholarly data and provide meaningful representations [?], but they focus on learning representations from meta-path will ignore the influences the same type of objects. Matrix factorization method based on multi-layered network [?] can also be adopted to scholarly data, while it has high computational cost when the scale of data becomes large.

Despite the significant performance in several network related tasks, network representations cannot well solve the recommendation task, since these representation methods care more about the pair-wise proximity rather than the ranking in recommendation problems. As a result, applying the above mentioned solutions to our problem will fail to capture the recommendation characteristics and deliver inappropriate results, which we will show in Section ??.

\section{B. Scholarly Data Recommendations}

In previous, content-based recommendation methods can provide accurate recommending results for industrial products [?]. However, utilizing relations among different objects, such as papers, authors, conferences and datasets, can better deal with the data characteristics and has been proved to obtain more effective recommendations in practice [?]. Due to the complexity of relations and objects, many scholarly recommendation methods first model the data as heterogeneous information networks [?]. Then, they use meta-path to generate a typical meta-path walk [?], like "author-paper-author" or "author-paper-dataset-paper-author", and obtain the pair-wise relationship between heterogeneous nodes in the network. Among all heterogeneous network based recommendation methods, a notable example of such approaches is TSR [?], a task guided, path-augmented network representation method which builds the paper and author representation in the same space using Bayesian Personalized Ranking (BPR) [?]. Similarly, HERec [?] uses the meta-path to represent the heterogeneous information network and makes the recommendation based on pair-wise ranking according to the metapath. Using attention mechanism in meta-paths also obtains competitive result in modeling scholar data [?]. However, meta-path based methods only focus on modeling relationships between nodes with heterogeneous representations but ignore 
the information propagation among homogeneous nodes (e.g., author relations and papers citations), which is extremely important for scholarly data. We allow homogeneous nodes in scholarly data connected, resulting in a multi-layered network, which contains $k$ types of nodes and the relationship between each type is deliverable.

\section{Dataset Retrieval}

Our problem is related but quite different from dataset retrieval. In recent years, dataset retrieval has attracted many industrial attentions. Companies, like Google, ResearchGate or Mendeley, begin to provide highly efficient dataset search engines [?]. The ease of accessing data enables scientists to build on others work, and providing scientists easier access to information and its provenance [?]. However, current dataset search engines focus on return datasets passively, when user offered a query. In modern research environment, a researcher may only be aware of a handful of datasets. Many useful datasets might easily be ignored by people in the searching period. As a result, actively recommending authors useful datasets and picking up possible missing ones will be a great complement for the whole research society.

\section{Methodology}

In this section, we present a novel representation model AMENDER for dataset recommendation. We first formulate the scholarly data as a type of heterogeneous information network and define the dataset recommendation problem, and then introduce the aggregation and attention mechanisms for learning the author and dataset embedding, which is next fed into a recommendation loss based on adversarial personalized ranking method. At last, a stable training algorithm, insensitive to data dependency, is proposed to optimize the recommendation loss.

\section{A. Preliminaries and Overview}

As illustrated in Fig. ??, a typical scholarly network can be modeled into a three-layered heterogeneous information network [?], in which intra-layer and inter-layer interactions are both taken in consideration.

Definition 1 (Scholarly Network): A scholarly network $G=\left(V, E, X_{p}\right)$ is a special kind of heterogeneous information network, which is comprised of three types of nodes $V=$ $\left(V_{a}, V_{p}, V_{d}\right)$ and four types of edges $E=\left(E_{a}, E_{p}, E_{a p}, E_{p d}\right)$, with subscription $a$ for authors, $p$ for papers and $d$ for datasets, as well as the additional features on paper vertices $X_{p}$.

In this scenario, a multi-layered network structure arises naturally from $G$ that consists of three sub-networks $G_{a}=$ $\left(V_{a}, E_{a}, \emptyset\right), G_{p}=\left(V_{p}, E_{p}, X_{p}\right)$ and $G_{d}=\left(V_{d}, \emptyset, \emptyset\right)$ along with author-paper and paper-dataset affinity matrices $E_{a p}$, $E_{p d}$. Following the denotation, we can formalize the studied problem as follows.

Problem 1 (Dataset recommendation based on scholarly network): Given an scholarly network $G$ and a set of authordataset preference records $S=\left\{\left\langle v_{a}, v_{d}\right\rangle\right\} \subseteq V_{a} \otimes V_{d}$ where $\left\langle v_{a}, v_{d}\right\rangle$ means that the author $v_{a}$ prefers the dataset $v_{d}$, our goal is to recommend the top- $\mathrm{k}$ relevant datasets for any $v_{a} \in V_{a}$.

To quantitatively measure the relevance of an author and a dataset and circumvent the data sparsity problem, we target on learning the representation for authors and datasets, denoted by $\mathbf{A} \in \mathbb{R}^{\left|V_{a}\right| \times d}$ and $\mathbf{D} \in \mathbb{R}^{\left|V_{d}\right| \times d}$, respectively, by fusing the graph structures and the paper content. The motivation is to characterize both authors and datasets in the same semantic space, where their proximity is measured regarding the latent research topics.

The overall framework is presented in Figure ??. Unlike many previous methods [?], [?] that only fuse the heterogeneous information by meta-path, our model will model both homogeneous and heterogeneous nodes interaction via two mechanisms: aggregation and attention. We will first obtain paper representation by aggregating the neighborhood papers, which will then be passed to authors and datasets through attention mechanism to obtain author and dataset representations. Finally, we learn the proximity of between authors and datasets by pair-wise ranking loss with a specially designed training procedure.

For the intra-layer of the scholarly network, aggregate mechanism can provide more useful information than directly leveraging paper contents. Meanwhile, the mean aggregation we used here is more computationally efficient for learning large network than other graph representation methods, including the attention approach. While for inter-layer information, the paper variance will be impactful in terms of the author and dataset representation. Using attention can better capture both the general information and paper importance comparing to aggregation.

\section{B. Aggregating the Paper Layer}

The intermediate layer including paper content and its relations can provide us abundant semantic information for our tasks. To build the semantic space where authors and dataset will be mapped, we adopt graph aggregation on the paper network to learn a latent space that captures the research topics.

Formally, given the paper network $G_{p}=\left(V_{p}, E_{p}, X_{p}\right)$, we learn the paper representation $\mathbf{P} \in \mathbb{R}^{\left|V_{p}\right| \times d}$, where $d$ is the dimension of representation vector, by the recent inductive attributed graph embedding model GraphSAGE [?]. For each paper $v_{p} \in V_{p}$, we obtain its representation by aggregating its immediate neighborhood paper information $K$ times. At $k$-th aggregation,

$$
\mathbf{P}_{v_{p}}^{k}=g^{k}\left(\operatorname{AGGREGATE}_{k}\left(\left\{\mathbf{P}_{v_{p}}^{k-1}\right\} \cup \mathbf{P}_{\mathcal{N}_{p p}\left(v_{p}\right)}^{k-1}\right)\right)
$$

where $\mathbf{P}_{\mathcal{N}_{p p}\left(v_{p}\right)}^{k-1}=\left\{\mathbf{P}_{v}^{k-1} \mid v \in \mathcal{N}_{p p}\left(v_{p}\right)\right\}, \mathbf{P}_{v_{p}}^{k-1}$ is the (k-1)-th aggregation of paper $v_{p}, \mathcal{N}_{p p}\left(v_{p}\right)$ is the immediate neighborhood of $v_{p}$, and $g^{k}$ is a non-linear function. Paper representation $\mathbf{P}$ is the output of the final aggregation, as $\mathbf{P}=\mathbf{P}^{K}$. And the initial representations $\mathbf{P}^{0}$ are generated from the paper's content features $X_{p}$. 


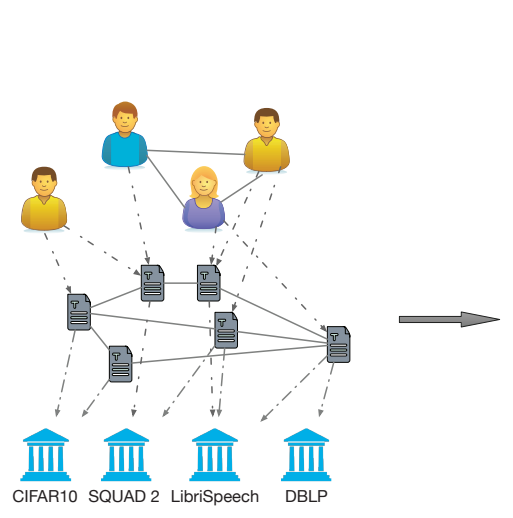

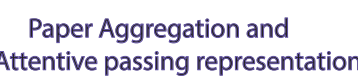

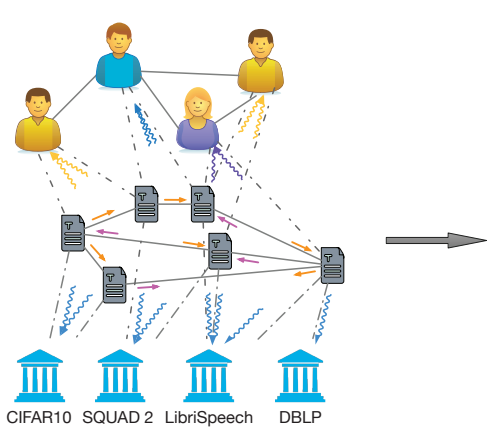

Merge author representation and

Author-Dataset relevance by APR Loss Recommend the Dataset

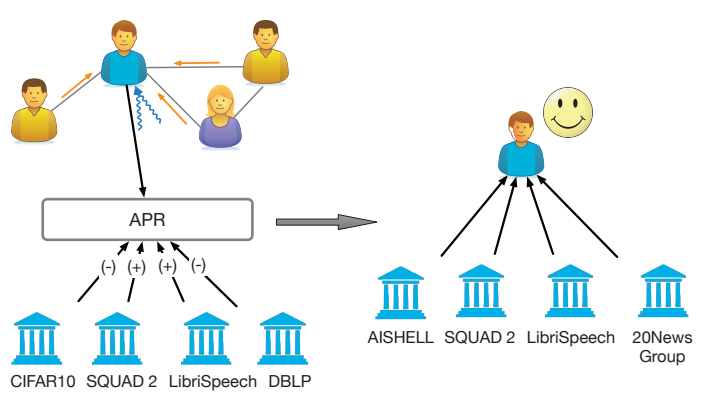

Fig. 2: The overview of AMENDER

Our results are not sensitive to the selection of aggregators. Here we adopt the simplest mean aggregation operator,

$$
\operatorname{AGGREGATE}_{k}(\cdot)=\sigma_{1}\left(\mathbf{W}_{p p}^{k} \cdot \operatorname{MEAN}(\cdot)\right) \text {, }
$$

where $\sigma_{1}$ is the ReLU activation function. The proposed aggregator is a linear approximation of a localized spectral convolution, which is proved in [?]. Lastly, the normalization is introduced to constrain the representation, which leads to the final result,

$$
\mathbf{P}_{v_{p}}^{k}=\frac{g^{k}\left(\operatorname{AGGREGATE}_{k}\left(\left\{\mathbf{P}_{v_{p}}^{k-1}\right\} \cup \mathbf{P}_{\mathcal{N}_{p p}\left(v_{p}\right)}^{k-1}\right)\right)}{\left\|g^{k}\left(\operatorname{AGGREGATE}_{k}\left(\left\{\mathbf{P}_{v_{p}}^{k-1}\right\} \cup \mathbf{P}_{\mathcal{N}_{p p}\left(v_{p}\right)}^{k-1}\right)\right)\right\|_{2}} .
$$

\section{Cross Layer Attention}

The paper representation $\mathbf{P}$ obtained in the previous section, along with author relationships and author-paper affinity (or dataset-paper affinity), is then utilized to learn the author representation (or dataset representations).

To learn author and dataset representation in the same semantic space with $\mathbf{P}$, we leverage the multi-head attention network [?] to link all the related papers to authors and datasets. The graph attention network component is a natural fit to learn inter-layer information propagation for it only computes the subgraph information, such as only author and his authored papers, rather than all instances in the training set as original attention structure [?]. Formally, for an author $v_{a} \in V_{a}$ (or a dataset $v_{d} \in V_{d}$ ), we will obtain its representation $\mathbf{A}_{v_{a}}^{(p)} \in \mathbb{R}^{d}$ (or $\mathbf{D}_{v_{d}}$ ) by passing the representation of all related papers $\mathcal{N}_{a p}\left(v_{a}\right)=\left\{v_{p} \mid v_{p} \in V_{p},\left(v_{a}, v_{p}\right) \in E_{a p}\right\}$ (or $\left.\mathcal{N}_{d p}\left(v_{d}\right)\right)$ using attention

$$
\begin{aligned}
& \mathbf{A}_{v_{a}}^{(p)}=\operatorname{ATTN}\left(\mathbf{P}, \mathcal{N}_{a p}\left(v_{a}\right)\right), \\
& \mathbf{D}_{v_{d}}^{(p)}=\operatorname{ATTN}\left(\mathbf{P}, \mathcal{N}_{d p}\left(v_{d}\right)\right) .
\end{aligned}
$$

For brevity, we will only illustrate the learning of $\mathbf{A}^{(p)}$ here, and building $\mathbf{D}^{(p)}$, i.e. $\mathbf{D}$, will follow the same process.

The attention mechanism will pass papers' representation to authors by first compute the attention coefficient between each related paper representation $\mathbf{P}_{v_{p \mid a}}, v_{p \mid a} \in \mathcal{N}_{a p}\left(v_{a}\right)$ and latest author's representation $\mathbf{A}_{v_{a}}^{(p)}$,

$$
\mathbf{e}_{v_{a}, v_{p \mid a}}=f\left(\mathbf{W}_{a p} \cdot \mathbf{A}_{v_{a}}^{(p)}, \mathbf{W}_{a p} \cdot \varphi\left(\mathbf{P}_{v_{p \mid a}}\right)\right),
$$

where $f$ is a 2-layer feedforward neural network parameterized by the non-linear LeakyReLU activation function, and $\varphi$ is a linear mapping function. The $\mathbf{e}_{v_{a}, v_{p \mid a}}$ measures the importance of paper $v_{p \mid a}$ to author $v_{a}$. To make coefficients comparable, we adopt softmax function for normalizing the attention coefficient,

$$
\alpha_{v_{a}, v_{p \mid a}}=\frac{\exp \left(\mathbf{e}_{v_{a}, v_{p \mid a}}\right)}{\sum_{v_{p} \in \mathcal{N}_{a p}\left(v_{a}\right)} \exp \left(\mathbf{e}_{v_{a}, v_{p}}\right)} .
$$

The normalized attention coefficients are then used to compute a combination of all related paper representations with ReLU activation $\sigma_{2}$ as the final output for each author,

$$
\operatorname{ATTN}\left(\mathbf{P}, \mathcal{N}_{a p}\left(v_{a}\right)\right)=\sigma_{2}\left(\sum_{v_{p \mid a} \in \mathcal{N}_{a p}\left(v_{a}\right)} \alpha_{v_{a}, v_{p \mid a}} \mathbf{W}_{a p}^{a t t n} P_{v_{p \mid a}}\right) .
$$

In addition, the multi-head strategy is applied to sample variant information from related papers and to make the learning more stable [?]. Specifically, we use $M$ independent attention mechanism $\left\{\operatorname{ATTN}^{m} \mid m=1, \cdots, M\right\}$ in our model. The final author representation is the multiplication between concatenated attention results and a parameter matrix $\mathbf{W}_{a p}^{\text {proj }} \in \mathbb{R}^{M d \times d}$ :

$$
\operatorname{ATTN}\left(\mathbf{P}, \mathcal{N}_{a p}\left(v_{a}\right)\right)=\|_{m=1}^{M} \operatorname{ATTN}^{m}\left(\mathbf{P}, \mathcal{N}_{a p}\left(v_{a}\right)\right) \cdot \mathbf{W}_{a p}^{p r o j},
$$

where $\|$ represents the concatenation.

\section{The AMENDER Framework}

1) Author Representation: In the above process, author representations $\mathbf{A}^{(p)}$ are obtained by using the paper representations. To integrate the author relationship that may contain extra information in the author network, aggregation is used again to refine the author representations, analogous to paper aggregation in Section ??, with initial $\mathbf{A}^{(a) 0}=\mathbf{A}^{(p)}$. For those authors have not published a paper, the mean vector of their neighborhood representation will be adopted 


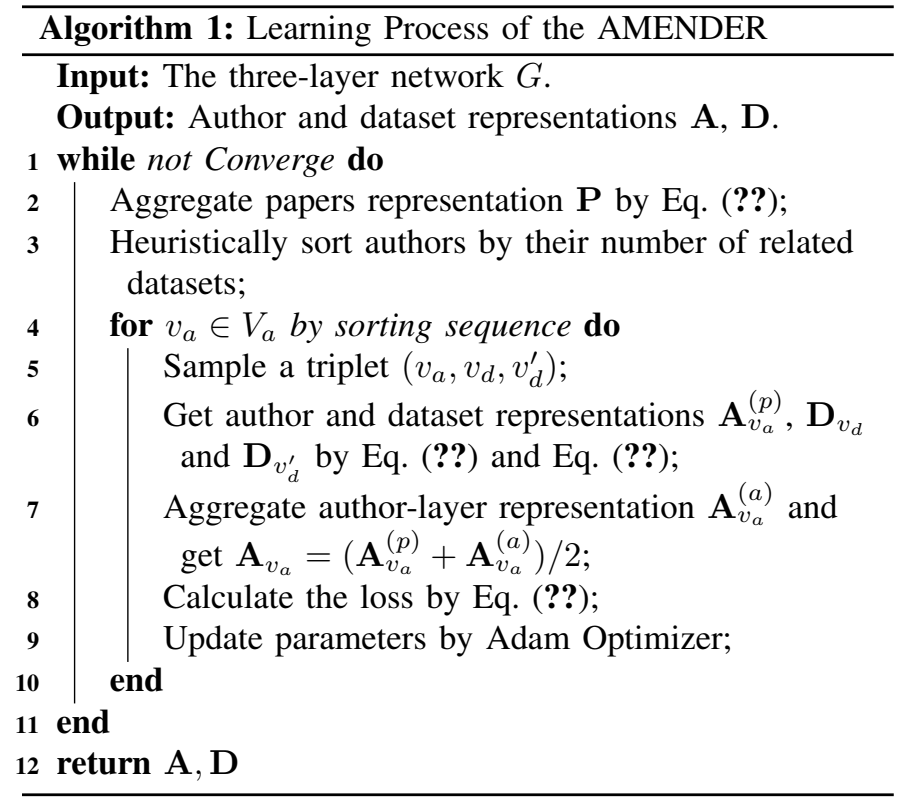

for prediction. Author representation from papers $\mathbf{A}^{(p)}$, and author neighborhood $\mathbf{A}^{(a)}$ are then combined for the final $\mathbf{A}=\left(\mathbf{A}^{(p)}+\mathbf{A}^{(a)}\right) / 2$.

To stabilize the author representation, we push $\mathbf{A}^{(p)}$ and $\mathbf{A}^{(a)}$ close by introducing a smooth loss,

$$
\mathcal{L}_{a a}=\sum_{v_{a} \in V_{a}} d\left(\mathbf{A}_{v_{a}}^{(p)}, \mathbf{A}_{v_{a}}^{(a)}\right),
$$

where $d$ is the pair-wise cosine similarity.

To the end, our objective function is defined as the combination of $\mathcal{L}_{a a}$ and $\mathcal{L}_{a d}$ :

$$
\mathcal{L}=\mathcal{L}_{a a}+\mathcal{L}_{a d} .
$$

2) Author and Dataset Proximity Loss: Given the representation $\mathbf{A}$ and $\mathbf{D}$ as well as the preference records $S$, where $S=\left\{\left\langle v_{a}, v_{d},\right\rangle \mid \exists v_{p} \in V_{p},\left(v_{a}, v_{p}\right) \in E_{a p},\left(v_{p}, v_{d}\right) \in E_{p d}\right\}$ in our model. We use the triplet personalized ranking loss function, derived from Bayesian Personalized Ranking (BPR) [?], to build the main loss for AMENDER. Denoting the negative samples by $S^{\prime}=\left\{\left\langle v_{a}, v_{d}^{\prime}\right\rangle \mid v_{a} \in V_{a}, v_{d}^{\prime} \in V_{d},\left\langle v_{a}, v_{d}^{\prime}\right\rangle \notin S\right\}$ we have the BPR loss

$$
\mathcal{L}_{a d}=\sum_{v_{a} \in V_{a}} \sum_{\substack{\left\langle v_{a}, v_{d}\right\rangle \in S \\\left\langle v_{a}, v_{d}^{\prime}\right\rangle \in S^{\prime}}} \ell\left(v_{a}, v_{d}, v_{d}^{\prime}\right)
$$

where

$$
\ell\left(v_{a}, v_{d}, v_{d}^{\prime}\right)=\left(1-\sigma_{3}\left(h\left(\mathbf{A}_{v_{a}}, \mathbf{D}_{v_{d}}\right)-h\left(\mathbf{A}_{v_{a}}, \mathbf{D}_{v_{d}^{\prime}}\right)\right)\right),
$$

where the $h$ is a distance metric (e.g., cosine distance in our experiments) and $\sigma_{3}$ is a non-linear mapping function to normalize the representation distance (e.g., sigmoid function).
3) Adversarial Personalized Ranking (APR): The traditional BRP method is vulnerable to adversarial noises [?]. When adding small noises to the recommendation items, the final representation might change severely. Recall the name ambiguity problem for the citation network, even the most advanced scholarship data sources will suffer from recognizing wrong authors [?]. Here we do not aim to propose a specific solution for this problem, but utilizing the adversarial personalized ranking loss to mitigate the impact of such a problem. As proposed in [?], we add the adversarial noises $\Delta$ to the author representations $\mathbf{A}$ only and build the loss:

$$
\mathcal{L}_{A P R}=\mathcal{L}+\lambda\left(\mathcal{L}_{a a, \Delta_{a d v}}+\mathcal{L}_{a d, \Delta_{a d v}}\right),
$$

where

$$
\Delta_{a d v}=\underset{\Delta,\|\Delta\| \leq \epsilon}{\operatorname{argmax}} \mathcal{L}_{a a, \Delta}+\mathcal{L}_{a d, \Delta},
$$

$\mathcal{L} \cdot, \Delta$ is the loss with adversarial noises added to author's representation and $\lambda$ is he adversarial regularization parameter.

\section{E. Training the Model}

1) Mini Batches: Due to the advantage of proposed model, for each author and dataset, only a subset of network need to be considered. For both aggregation and attention layer, the operation will agnostic to the neighborhood nodes considered. Benefiting from such homogeneity, we can easily apply mini-batch learning here for efficient training. As shown in Algorithm ??, we will sample pairs of negative and positive author-dataset instances to be a mini-batch. Then we optimize our model w.r.t. the samples. Such method will cost few computational resources and runs faster comparing to full batching trainings.

2) SortaGrad: Motivated from the previous successful deep learning models [?], we adopt a simple curriculum learning strategy to train our network, SortaGrad. The training process is provided in Algorithm 1. SortaGrad is previously used in the speech recognition field to stable the training procedure and solve the product dependency issue. Here we adopt the strategy for efficient training and to deal with the dependency problem of related neighbors. The number of related datasets is used as a heuristic for difficulty, because authors who used too many datasets are harder to indicate the representation than those who used less.

\section{EXPERIMENTS}

We empirically studied the effectiveness of proposed method on a real dataset. We first describe the dataset and then present our results.

\section{A. Datasets}

Our experiment is conducted on the records collected from a dataset search engine called Delve ${ }^{1}$, covering academic papers and datasets mostly from artificial intelligence domains like machine learning, data mining and computer vision, etc. In our experiment, the network we used contains 73,855 authors,

\footnotetext{
${ }^{1}$ http://adatahub.com
} 
79,775 papers and 6,863 datasets in total. Here we treat the citation network as paper relationships, co-author network as the author relationships (they may have co-authored on other papers out of this collection). In total, there are 191,096 authorpaper edges and 15,572 paper-dataset edges. The network is sparse, as only around $14 \%$ of authors have used one or more dataset, and the others do not have direct links to any datasets we collected.

\section{B. Compared Methods}

We extend 11 related state-of-the-art methods that span three categories to our problem for comparison:

1) Network Representations: Network representation methods learn the representations for author, paper and datasets for general purposes. We select homogeneous model Node2Vec [?], distributional representation model Graph2Gaus [?], heterogeneous model Metapath2Vec [?] and multi-layered network model MANE [?]. Note that MANE is highly memory demand, and we can only learn two layers, author and datasets, on our 128 Gigabytes machine.

2) Ranking based Recommendations: Ranking based recommendation methods use the ranking loss to learn the relevance between authors and datasets. Here we select a baseline Matrix Factorization method, contentbased model BPR [?] and WARP [?], and heterogeneous information network based model HERec [?].

3) Social Recommendation: Social recommendation methods leverage author relationships for promoting recommendation performance. We select two state-ofthe-art social recommendation models TSR [?] and CSE [?].

4) Proposed Method: One may be interested in how important the role of the intermediate layer of papers is playing in our model, because a simple operation can form an author-dataset bipartite network by linking an author and a dataset if the dataset is used by author. We evaluate our proposed model on this setting by filtering out the paper information, notated AMENDER , and compare it with the original model AMENDER.

For fair comparison, we set representation dimension $d=$ 64 for all methods. For all the state-of-the-art comparison methods, we set all the hyper parameters the same as reported in the original papers. In the proposed method, the adversarial regularization parameter $\lambda$ is set as 0.2 , and the number of multi-head attention $M$ is set as 3 for the best performance. Paper content vectors are pre-trained using all abstracts via doc2vec [?]. The detailed parameter sensitivity discussion will be described in section ??.

\section{Recommendation to Existing Authors}

We first evaluate our model's effectiveness by recommending datasets to existing authors. In this experiment, each user in the test set will be randomly masked a few datasets, and we will predict whether a user will use the masked datasets. Here we formalize the problem as pair-wise ranking orders between correlated and uncorrelated author-dataset pairs. We create three test sets that contain 50\%, 20\% and 5\% randomly selected correlated author-dataset pairs and equal number of randomly selected uncorrelated pairs. By convention, we report both the AUC (Area Under ROC Curve) score and AP (Average Precision) score to evaluate how highly ground-truth correlated datasets have been ranked over other datasets.

The performances of all methods are presented in Table ??. We can observe that AMENDER consistently outperforms all others at all the ratio of training and testing set. Specifically, AMENDER can obtain about 0.93 AUC with only $50 \%$ training samples, which is higher than many methods using $95 \%$ for training. AMENDER paper $_{\text {also performs worse than }}$ AMENDER, which indicates that our model can better capture author-dataset correlation with the supplement of papers.

Comparing to many network embedding methods, such as Graph2Gauss, MetaPath2Vec, etc, AMENDER obtains better performance because it can discriminate different types of nodes and learns the representation in different semantic space. AMENDER can compare the dataset representation with author representation directly, and obtain the recommendation result. Comparing to some user-item recommendation methods, AMENDER obtains better performance because it utilize the information from different layers. It is worth mentioning that HERec can obtain a higher performance than other recommendation methods, because it considers the metapath relations and learns the representation by also considering different layers' information. What's more, it is interesting that Graph2Gauss obtains the second best performance (better than recommendation methods), probably because the objective of network representation methods is pair-wise distance, which totally fits the pair-wise comparison task. The recommendation methods, on the other hand, ignores the pair-wise ranking information due to their loss functions. While the AMENDER utilizes the author-dataset pair-wise ranking as the learning object so that obtains the best performance.

\section{Cold Start Recommendations}

A more realistic yet challenging setting is to infer nonexisting user's preference on datasets. For example, young students have no published papers but only linked with their advisors. In this experiment, we randomly selected ten percent of authors with few links and masked all their used datasets and their published papers in both training and testing phase. In the testing phase, when predicting the possible datasets to one of these authors, only the authors relations in training set are used. In this experiment, there are many baseline methods that cannot deal with the cold start problem easily, such as Graph2Gauss, Node2Vec, MF, MANE, etc. For these methods, we use the mean vector of all related authors representations as the target author's representation vector, and make the recommendation based on it. Here we use the Recall@k, precision@ $k$ and corresponding F1-score for evaluating the recommendation correctness.

Table ?? shows the performance of all models at different setting of $k$. We find that network representation methods 
TABLE I: Recommendation accuracy to existing authors (the best results in bold, while the second best with $\mathrm{a} *$ )

\begin{tabular}{|c|c|c|c|c|}
\hline \multirow{2}{*}{ Methods } & \multirow{2}{*}{ Metrics } & \multicolumn{3}{|c|}{ Training Percentage } \\
\hline & & $50 \%$ & $80 \%$ & $95 \%$ \\
\hline \multirow{2}{*}{ Node2Vec } & AUC & 0.844 & 0.890 & 0.901 \\
\hline & $\mathrm{AP}$ & 0.802 & 0.859 & 0.870 \\
\hline \multirow{2}{*}{ Graph2Gauss } & $\overline{\mathrm{AUC}}$ & $0.891^{*}$ & $0.918^{*}$ & $0.932^{*}$ \\
\hline & $\mathrm{AP}$ & $0.884^{*}$ & $0.908^{*}$ & $0.925^{*}$ \\
\hline \multirow{2}{*}{ MANE } & AUC & 0.863 & 0.878 & 0.885 \\
\hline & AP & 0.855 & 0.868 & 0.872 \\
\hline \multirow{2}{*}{ MetaPath2Vec } & $\overline{\mathrm{AUC}}$ & 0.838 & 0.864 & 0.854 \\
\hline & $\mathrm{AP}$ & 0.860 & 0.886 & 0.884 \\
\hline \multirow{2}{*}{ MF } & AUC & 0.714 & 0.750 & 0.768 \\
\hline & $\mathrm{AP}$ & 0.774 & 0.816 & 0.836 \\
\hline \multirow{2}{*}{ BPR } & AUC & 0.821 & 0.862 & 0.881 \\
\hline & $\mathrm{AP}$ & 0.796 & 0.851 & 0.888 \\
\hline \multirow{2}{*}{ WARP } & AUC & 0.828 & 0.878 & 0.881 \\
\hline & AP & 0.798 & 0.869 & 0.875 \\
\hline \multirow{2}{*}{ HERec } & AUC & 0.866 & 0.917 & 0.922 \\
\hline & $\mathrm{AP}$ & 0.826 & 0.891 & 0.900 \\
\hline \multirow{2}{*}{ TSR } & AUC & 0.824 & 0.846 & 0.887 \\
\hline & $\mathrm{AP}$ & 0.818 & 0.839 & 0.868 \\
\hline \multirow{2}{*}{ CSE } & AUC & 0.826 & 0.885 & 0.901 \\
\hline & $\mathrm{AP}$ & 0.794 & 0.875 & 0.891 \\
\hline \multirow{2}{*}{ AMENDER } & $\overline{\mathrm{AUC}}$ & 0.881 & 0.892 & 0.910 \\
\hline & $\mathrm{AP}$ & 0.866 & 0.878 & 0.900 \\
\hline \multirow{2}{*}{ AMENDER } & AUC & 0.933 & 0.945 & 0.955 \\
\hline & $\mathrm{AP}$ & 0.925 & 0.940 & 0.951 \\
\hline
\end{tabular}

cannot perform well when addressing this recommendation problem. The only effective network embedding method is Metapath2Vec, which is good at learning heterogeneous representations. This is mainly caused because many representation methods cannot provide an effective representation for the cold-start problem.

Recommendation based methods perform well in this experiment. For example, HERec obtains the second best performance among almost all settings of recall@ $k$, showing the effectiveness of incorporation extra layer for recommendation. Also, the BPR can obtain the second best performance when precision and F1 score at top three recommendations, and achieves generally good performance comparing to other methods at all recall and precision scenarios. Furthermore, comparing the difference between this experiment and the recommendation to existing authors, representation based methods performance changed dramatically. This is because that only using pair-wise relationship as objective cannot fit for the recommendation task, the ranking loss like BPR is more effective, especially in the recommendation task like selecting candidates.

In this experiment, AMENDER again has the best performance, and obtain about $10 \%$ relative recall gain when only a few candidate datasets are given. Because AMENDER can better combine the BPR loss and representation advantage together, also a better manipulating information from different types of nodes can help build the recommendation method more effectively.

\section{E. Parameter Sensitivity}

In this section, we discuss the sensitivity of several important parameters in our model, Adversarial parameter $\lambda$, the number of heads $M$ and dimension of representation $d$ and the effect of APR in training.

1) Adversarial Parameter $\lambda$ : Figure ??) generally shows a stable performance of AMENDER when varying $\lambda$. However, when $\lambda$ is 0.2 , the model obtains the best performance. It is interesting to see that with a higher $\lambda$, the performance gets a little worse. This is probably due to the noises raised by adversarial attack, as reported in [?].

2) \#Heads of Multi-head Attention: For a fair comparison, we fixed the dimension of embedding $d$ to be 64 and analyze the sensitivity of the number of head used for the multi-head attention. The results are presented in Fig. ??. We can see that AMENDER benefits from the multi-head structure that the AUC rises when the number of heads increases. However, when the number of heads is larger than 3 , the prediction performance decreases, caused by potential overfitting.

3) Representation Dimension $d$ : The result w.r.t. $d$ is illustrated in Fig. ??. We can see that AMENDER obtains the best representation when the dimension reaches 64 . When $d$ gets larger than 64, the AUC score drops a little and stays fluctuate due to data variance.

4) Effect of Adversarial Noise: As described in section ??, we used APR to enhance the robustness of representation learning. Figure ?? illustrates that adding adversarial noises in the training phase can significantly improve the robustness of representation and finally delivered better results, comparing to the case only using BPR.

\section{F. Topics of Datasets and Authors from their Representation}

To interpret the learned dataset and author representation, we visualize the obtained author and dataset embedding, $\mathbf{A}$ and $\mathbf{D}$, in 2-dim space by t-SNE [?] approach. Due to the space limit, we randomly selected 100 authors (triangles) and 100 datasets (squares) and show them in Figure ??. Also, we apply $k$-means to cluster the representations and show a word-cloud for interpreting each cluster ${ }^{2}$. Here we select $k$ equals to four, due to the selection of Silhouette Coefficient [?]. As presented in the figure, each cluster has different keywords. For example, the cluster with blue color is more related to machine learning and neural networks research, and green cluster is more about pure clustering or classification studies. Meanwhile, the orange cluster is about system performance, while red cluster is about data mining. It is interesting that system performance and data mining have an overlapping territory, indicating they may have closer relations. To investigate further, we zoom in the overlapping area, and found that most of the authors and datasets in that area are related to database performance and theory. This is reasonable because the database serves as infrastructures for data mining. Meanwhile, the efficiency and scalability of

\footnotetext{
${ }^{2}$ Each cluster is interpreted by the paper contents published by the group of authors, and the papers citing the datasets.
} 
TABLE II: Recall, Precision and F1-Score@ $k$ for cold start users (the best results in bold, while the second best with a $*$ )

\begin{tabular}{|c|c|c|c|}
\hline \multicolumn{2}{|c|}{ Methods } & (a) 3 & @5 \\
\hline \multirow{4}{*}{\multicolumn{2}{|c|}{$\begin{array}{c}\text { Node2Vec } \\
\text { Graph2Gauss } \\
\text { MANE } \\
\text { MetaPath2Vec }\end{array}$}} & 0.235 & 0.267 \\
\hline & & 0.202 & 0.242 \\
\hline & & 0.241 & 0.273 \\
\hline & & 0.584 & 0.629 \\
\hline \multicolumn{2}{|c|}{$\mathrm{MF}$} & 0.480 & 0.521 \\
\hline \multicolumn{2}{|c|}{ BPR } & 0.615 & 0.661 \\
\hline \multicolumn{2}{|c|}{ WARP } & 0.621 & 0.668 \\
\hline \multicolumn{2}{|c|}{ HERec } & 0.626 & $0.672^{*}$ \\
\hline \multicolumn{2}{|c|}{ TSR } & $0.637^{*}$ & 0.657 \\
\hline \multicolumn{2}{|c|}{ CSE } & 0.605 & 0.648 \\
\hline \multirow{2}{*}{\multicolumn{2}{|c|}{$\begin{array}{c}\text { AMENDER } \\
\text { AMENDER }\end{array}$}} & 0.587 & 0.655 \\
\hline & & 0.738 & 0.784 \\
\hline \multicolumn{4}{|l|}{1.000} \\
\hline \multicolumn{4}{|l|}{0.975} \\
\hline \multicolumn{4}{|l|}{0.950} \\
\hline \multicolumn{4}{|l|}{ 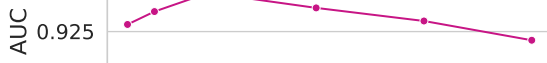 } \\
\hline \multicolumn{4}{|l|}{0.900} \\
\hline \multicolumn{4}{|l|}{0.875} \\
\hline 0.850 & 0.2 & 0.4 & 0.8 \\
\hline
\end{tabular}

(a) Adversarial Parameter $\lambda$

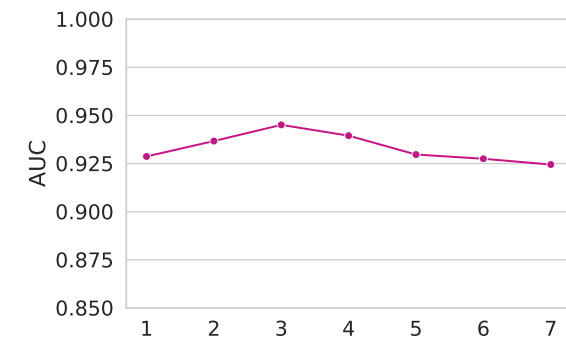

(b) \# of heads

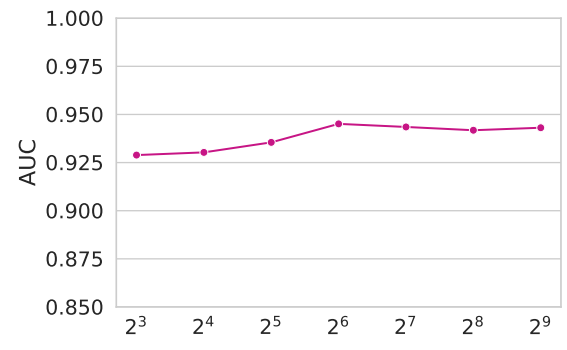

(c) Representation dimension $d$

Fig. 3: Parameter Sensitivities (a) Adversarial regularization parameter $\lambda$. (b) The number of heads used for multi-head attention. (c) The representation dimension $d$.

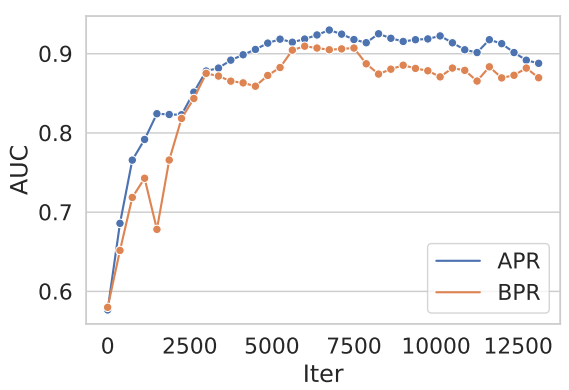

Fig. 4: The effectiveness of APR on representation robustness.

database is a heated topic for system field. The researchers studying about database area will certainly use dataset in both data mining and system area. For example, professor Xiaodong Zhang, from Ohio state university, has research interests mainly focus on "data management in scalable system". Also, Michaela Götz, a former Ph.D. student at Cornell University, has published her most influential paper in SIGMOD, a top conference in database theories. These author names can be found in the overlapping area. Datasets in this area are also interesting to discover. For example, both TREC-1 and TREC-3 (very closely located) are from the Text REtrievel Conference (TREC). They contain billions of webpages and often serve as datasets for query performance evaluation and text mining. Also, the CNN Transcripts contain a large corpus of CNN transcripts. The dataset is constantly used for text classification or the performance benchmarks for information retrieval. According to the interesting visualization results, we confirm that the learned representations are meaningful and well capture the relations between authors and datasets.

\section{G. Case Study}

In this section, we empirically illustrated how our model can be used in the real-world recommendation tasks for two categories. In one category, influential researchers in our crawled subgraph, especially in machine learning and data mining field, like Jure Leskovec, or Yoshua Bengio, have used dozens of datasets. We will find out more related datasets based on their previous research and recommend to them as compensation. In the other category, young talented researchers, like Yuxiao Dong and Xiang Ren, only used a few datasets in our scholarly network. Recommending useful datasets for them based on their social relations can help them to explore new areas. The experimentally studied cases are illustrated in TABLE ??.

1) Recommending for existing users: We first analyze the recommendation results for Jure Leskovec, a famous professor studying graph mining and data mining at Stanford University. In the training phase, we randomly masked a few datasets and 
TABLE III: Case Study for several researchers (Jure Leskovec as an existing user, while Xiang Ren and Yuxiao Dong as cold start users). Notations $\checkmark$ represents correctly recommended; $\square$ represents correct yet not recorded; $\times$ represents never used.

\begin{tabular}{|c|c|c|c|c|}
\hline Author & Dataset In Training & Dataset Recommended & Rank & Used \\
\hline \multirow{6}{*}{ Jure Leskovec } & \multirow{6}{*}{$\begin{array}{c}\text { Google Web Graph: Webgraph from Google } \\
\text { ca-GrQc: Collaboration network in Arxiv } \\
\text { Enron Network: Enron Email Network } \\
\text { Kronecker: Kronecker Graph Generator } \\
\text { LiveJournal SN: Online Blogging Community } \\
\ldots . . .\end{array}$} & Flickr Dataset (Social Relations) & 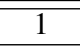 & 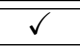 \\
\hline & & protein-protein interaction (PPI) & 2 & $\checkmark$ \\
\hline & & Reality Mining cell-phone & 3 & $x$ \\
\hline & & Facebook Dataset (About Social Relations) & 4 & $\checkmark$ \\
\hline & & Call Data Records (CDR) & 5 & $\square$ \\
\hline & & ..... & & \\
\hline \multirow{3}{*}{ Xiang Ren } & \multirow{3}{*}{ None } & NewsBank: News Article Dataset & 1 & $\checkmark$ \\
\hline & & Huffington Post, Online News Service & 2 & $\checkmark$ \\
\hline & & ASRS (Aviation Safety Information Dataset) & 3 & $x$ \\
\hline \multirow{3}{*}{ Yuxiao Dong } & \multirow{3}{*}{ None } & Reciprocal Link: About how links formed & 1 & $\checkmark$ \\
\hline & & Epinions: Online Social Product Review & 2 & $\checkmark$ \\
\hline & & IMDB Dataset: e Internet Movie Database & 3 & $\square$ \\
\hline
\end{tabular}

\section{Author and Dataset Representation}

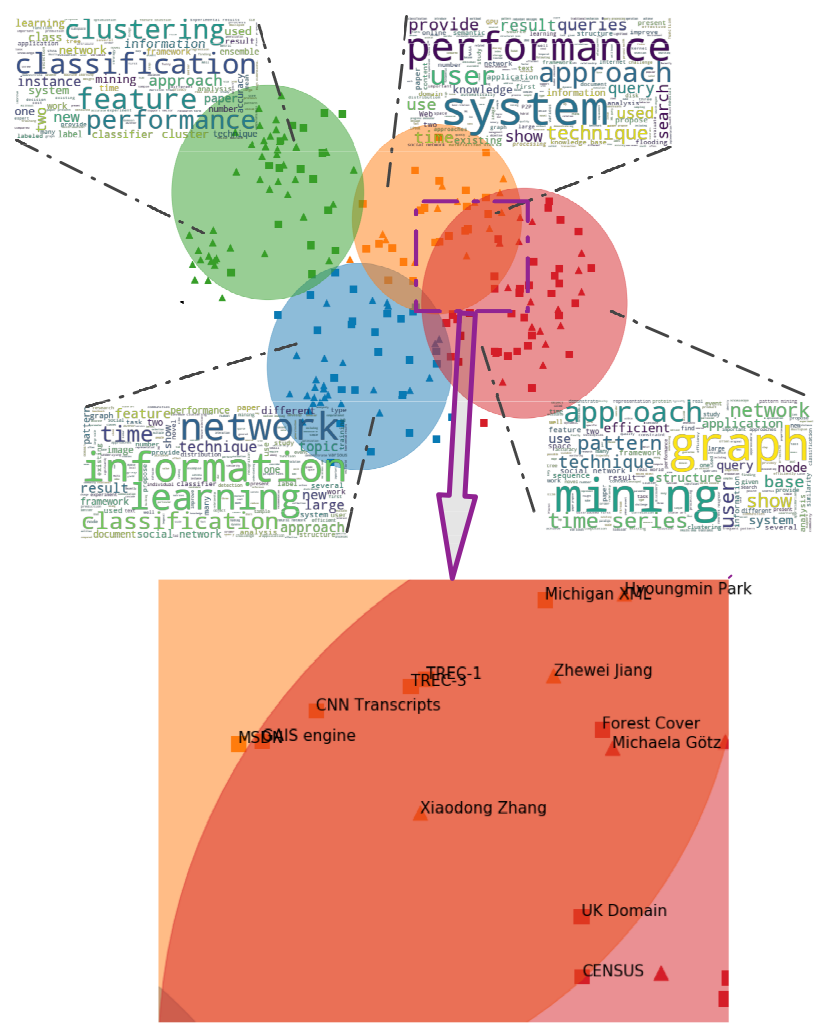

Fig. 5: Visualize author (triangle) and dataset (square) representation. Clusters are interpreted by the word-cloud of research topics.

publications Jure had. In the inference phase, we rank the possible datasets he would use excluding those datasets used in the training phase. For example, Google web graph contains the subgraph served in google, other datasets, like Enron Email, caGrQc, LiveJournal social network, are all social networks recording human interactions. Kronecker is not a graph dataset, but it is a functioning tool to generate a random graph automatically. osed. As listed in TABLE ??, most of the datasets used for training Jure Leskovec's representation are about web mining and graphs, which matches Jure's research interest in graph mining $\mathrm{q}$ In the inference phase, we collect the top five datasets recommended: the Flickr, PPI, Reality Mining Cellphone, Facebook, and Call Data Records. For all five datasets recommended, four of them were truly used by Jure in his other research works. More specifically, three datasets, Flickr, PPI and Facebook, are all recorded in our scholarly network and were masked in the training phase. Meanwhile, two datasets about cellphone services are recommended but not used according to our scholarly network. The Call Data Records, which ranked the fifth, is a large cellular network, recorded voice, data, and SMS services. We find that Jure Leskovec used the Call Data Records (CDR) dataset in other papers beyond our scholarly network [?]. While the Reality Mining Cellphone dataset, ranked the third, is also a cellphone call graph network and is very similar to Call Data Records. Jure has never used the Reality Mining Dataset, but there are two reasons why Reality Mining Dataset is a reasonable recommendation. First, Jure Leskovec's Ph.D. advisor, Christos Faloutsos, used the Reality Mining Cellphone dataset several times. The close coauthor-ship and research interests between Jure and Christos may indicate that they will share the same interest about certain datasets. Second, Reality Mining is a large complex graph dataset, which matches Jure's research interests very well.

2) Recommending for Cold Start users: In this experiment, we remove the authors' entire publications relations as well as the used datasets in the training phase, and then recommend the dataset by coauthorships only. We take Dr. Yuxiao Dong and Prof. Xiang Ren as examples. In our scholarly network, they only have linked to less than ten papers and used about three or five datasets in total.

As presented in TABLE ??, AMENDER obtained great recommendations for Prof. Xiang Ren. For example, we precisely recommended the NewsBank and Huffington Post datasets, which are always used for text mining and key phrase extraction problems. ASRS, an aviation Safety information dataset, is recommended because it is also a large text mining corpus and often used for keyword extraction methods. Though Xiang 
Ren did not use the ASRS dataset directly, Xiang Ren's active coauthor Jiawei Han used the dataset many times and Xiang has also cited papers using ASRS dataset for keyword extraction tasks. The recommendation for Yuxiao Dong is also effective. Dr. Yuxiao Dong has published many papers about social network and graph mining. The recommended datasets like Reciprocal links and IMDB are constantly used for graph mining tasks and used by Yuxiao as well. Epinion network, ranked on the second, is an online product review dataset and is often used for bipartite network studies. Though we did not record that Yuxiao used Epinion dataset in our scholarly network, he used this dataset in his later publications [?].

\section{CONCLUSION}

In this paper, we studied the problem to recommend appropriate datasets for authors. We designed AMENDER to tackle the relevance measurement between authors and datasets. AMENDER can better leverage both intra-layer and interlayer information, and can better model the author-dataset relationships by utilizing the adversarial ranking loss, comparing to the solutions that extend existing heterogeneous network embedding models and social recommendation models. We

plan to deploy our model and benefit researchers in AI domain in the future. 